\title{
Effects of Heavy-Atom Substituents on Matrices Used for Matrix-Assisted Laser Desorption-Ionization Mass Spectrometry
}

\author{
Lisa M. Preston-Schaffter, Gary R. Kinsel, and D. H. Russell \\ Texas A \& M University, Department of Chemistry, College Station, Texas USA
}

\begin{abstract}
The data reported show that heavy-atom substitution on matrices that are used for matrix-assisted laser desorption-ionization mass spectrometry enhance $[\mathrm{M}+\mathrm{H}]^{+}$ion yields of both Leu-enkephalin and vitamin $B_{12}$. Heavy-atom substitution alters the excited state relaxation dynamics of the matrices as indicated by measured decreases in the measured luminescence (fluorescence and /or phosphorescence) observed for heavy atoms that contain matrices relative to the nonheavy-atom-substituted matrices. The results presented are consistent with the direct involvement of an electronically excited state of the matrix in the formation of analyte $[\mathrm{M}+\mathrm{H}]^{+}$ions. ( $(J$ Am Soc Mass Spectrom 1994, 5, 800-806)
\end{abstract}

S uccessful ionization of any analyte by matrix-assisted laser desorption-ionization (MALDI) [1, 2] requires the proper choice of matrix, laser wavelength, matrix-to-analyte $(\mathrm{M}-\mathrm{A})$ ratio, and other experimental parameters. The method of choosing the matrix is guided by the simplest of empirical considerations, that is, that the organic matrix must absorb at the wavelength of the desorbing laser. However, because the desired result from the MALDI experiment is the production of an ionic form of the intact analyte, the matrix also must be capable of promoting electron transfer, proton transfer, or cation attachment to the analyte. Although several models for the desorption [3-6] and ionization [7-10] processes involved in MALDI have been proposed, numerous questions about the mechanism of ion formation remain unanswered. One question of particular interest is the role of the matrix in MALDI. In the simplest terms the matrix serves two purposes:

1. The matrix provides a means for direct energy deposition into the matrix-analyte solid via photon absorption.

2. The matrix provides a source of protons to form the $[\mathrm{M}+\mathrm{H}]^{+}$ion of the analyte.

Experiments by Levis and Romano [11, 12] suggest that desorption and ionization are separate processes, that is, the analyte can be volatilized as an intact neutral via photon absorption by the matrix. Speir and Amster [13] also have shown that conditions can be tailored to promote desorption of neutral peptides by

Address reprint requests to Professor David H. Russell, Department of Chemistry, Texas A \& M University, College Station, TX 77843-4719. using the same matrices that are used to produce ions. Specifically, Speir and Amster showed that segregation of the matrix and analyte in the solid state facilitates the desorption of the neutral analyte.

Because analyte $[\mathrm{M}+\mathrm{H}]^{+}$ions are formed by a proton transfer reaction that involves the matrix and analyte, we argue that the best framework to describe the formation of positive ions is in terms of an acid-base reaction, where the matrix acts as a proton donor and the analyte acts as a proton acceptor. Our working hypothesis for ion formation in MALDI is that UV photon absorption produces excited state "acidic" matrix species [designated (matrix)*] that undergo proton transfer to a "basic" analyte to form [M $+\mathrm{H}]^{+}$ ions [9]. This hypothesis is supported by the fact that MALDI matrices (for a review of MALDI matrices, see [14]), for example, 4-nitroaniline and sinapic acid, are generally aromatic amines or alcohols that are known to be more acidic in the excited state than in the ground state [15-17]. Although electronically excited states of a matrix can relax rapidly by radiative and nonradiative pathways (internal conversion, intersystem crossing, and/or proton transfer [18, 19]), strongly coupled matrix-analyte interactions (specifically hydrogen bonding) may facilitate proton transfer on the time scale of radiative relaxation $\left(10^{-9} \mathrm{~s}\right)$. It should be emphasized that (matrix)* does not have to be the $S_{1}$ excited state of the matrix. Vibrationally excited ground state species (formed by internal conversion) or triplet states (formed by intersystem crossing) [20] could lead to proton transfer on the $10^{-6}$ - [sampled by time-offlight (TOF) instruments] or $10^{-3}-\mathrm{s}$ [sampled by Fourier-transform ion cyclotron resonance (FTICR)] time scale. Isotopic labeling experiments could provide valuable insight about the specific proton transferred [21]; however, the proton(s) of acidic or basic func- 
tional groups $\left(\mathrm{NH}_{2}, \mathrm{OH}, \mathrm{COOH}\right)$ are highly labile and subject to rapid exchange that could mask the desired information.

Mechanisms that involve reactions other than excited state proton transfer have been invoked to explain MALDI. Ehring et al. [8] proposed a radical cation as the initiator for proton transfer reactions, and Wang et al. [10] proposed that proton exchange takes place from a "photochemically activated" matrix to the analyte to produce $\left[\mathrm{M}+\mathrm{H}^{+}\right.$ions. Chiarelli et al. [7] reported evidence that suggests that excited state proton transfer as a solid-state ionization mechanism is applicable to both laser desorption-ionization of small organics as well as MALDI. Although other groups have suggested the involvement of excited state proton transfer reactions in gas-phase ion chemistry [7, 22] and ionization processes, none of these studies has attempted to correlate excited state relaxation dynamics with the MALDI mass spectrometry experiment. One means to test the involvement of excited state processes in MALDI is to perturb the excited state chemistry of a matrix and observe the effect on analyte $[\mathrm{M}+\mathrm{H}]^{+}$ion yield. Heavy-atom substitution $(\mathrm{Br}$, I, $\mathrm{Cl}, \mathrm{F}$ ) of aromatic organic molecules is known to alter excited state relaxation dynamics by promoting intersystem crossing to form triplet species [23, 24]. The experiments described herein use matrix pairs (two structurally similar aromatic compounds: one with a heavy-atom substituent and one without) to probe the correlation between matrix relaxation dynamics [as illustrated by measured changes in matrix luminescence (fluorescence and/or phosphorescence)] and MALDI analyte $[\mathrm{M}+\mathrm{H}]^{+}$ion yields.

\section{Experimental}

\section{Mass Spectrometry}

A 1.0-m linear time-of-flight mass spectrometer, designed and built in this laboratory, is used for the MALDI experiments. The ion source and acceleration region consists of three acceleration plates and two sets of steering electrodes. The first plate is biased at +10 $\mathrm{kV}$ and the second and third plates are held at ground. Ions are detected by using a standard dual microchannel plate detector biased at $-2.25 \mathrm{kV}$ and positioned at the end of the $1.0-\mathrm{m}$ flight tube. The transient ion signal is collected by a $50-\Omega$ impedance-matched 350 $\mathrm{MHz}$ oscilloscope (LeCroy 9420, LeCroy, Chestnut Ridge, NY) that is interfaced to a microcomputer. Time-of-flight mass spectra are calibrated and analyzed with the GRAMS386 commercial software package (version 1.04 from Galactic Industries Corp.).

A Laser Photonics sealed-cartridge nitrogen laser (337-nm output, Model LN300) is used to generate ions. The laser has a 5-ns pulse width and is operated at a repetition rate of $4 \mathrm{~Hz}$. Laser power is controlled with a mechanical iris and is kept constant throughout all experiments to minimize power fluctuations due to changes in spot size. The laser beam is focused to a line of approximately $3.0 \times 0.5 \mathrm{~mm}$ on the probe tip surface at a $50^{\circ}$ incident angle. Focusing is achieved by using a spherical lens (1-m focal length) to collimate the 337-nm laser beam and a spherical lens (200-mm focal length) to obtain the final narrow line. The laser power density used in these experiments, calculated from energy measurements taken with a Vector D200 (Scientech, Boulder, $\mathrm{CO}$ ) energy meter, is approximately $10^{6} \mathrm{~W} / \mathrm{cm}^{2}$.

\section{MALDI Sample Preparation}

Vitamin $B_{12}$ and Leu-enkephalin were purchased from Sigma Chemical (St. Louis, MO) and used without further purification. The organic compounds 2-bromo4,6-dinitroaniline, 4-hydroxy-3-nitrobenzoic acid, 5iodosalicylic acid, 5-bromosalicylic acid, 5-chlorosalicylic acid, 5-fluorosalicylic acid, salicylic acid, 2,4-dihydroxybenzoic acid, 2,4-dinitroaniline, and 5bromo-2,4-dihydroxybenzoic acid monohydrate were purchased from Aldrich Chemical Co. (Milwaukee, WI). 4-hydroxy-3-iodo-5-nitrobenzoic acid was purchased from Sigma Chemical Co. (St. Louis, MO). These compounds were used as MALDI matrices without further purification. All matrix solutions were prepared in methanol at a concentration of $0.11 \mathrm{M}$. Analyte solutions were prepared at a concentration of 0.93 $\mathrm{mM}$ in methanol.

The MALDI samples were prepared by first coating the sample probe tip with $2 \mu \mathrm{L}$ of a $5-\mathrm{mg} / \mathrm{mL}$ solution of nitrocellulose (purchased from Schleicher and Schuell). Nitrocellulose was used to improve the reproducibility of MALDI analyte ion yields [25]. The thin film of nitrocellulose was dried and $3 \mu \mathrm{L}$ of premixed (50:50 v/v) matrix and analyte solution were deposited onto the sample probe tip and allowed to air dry. The optimum M-A ratio obtained by using both the heavy-atom-substituted and nonsubstituted matrices was approximately 120:1 for Leu-enkephalin and vitamin $B_{12}$. This value was determined by testing a number of $M / A$ ratios (20:1 $\rightarrow$ 2000:1) and selecting the one that produced the highest abundance of $[\mathrm{M}+$ $\mathrm{H}^{+}$analyte ions for each matrix. All reported spectra are the result of averaging ion signals from 100 laser shots.

\section{Absorption and Luminescence Measurements}

Absorption spectra $(245-550 \mathrm{~nm})$ were acquired with a Lambda $3 b$ Perkin-Elmer (Norwalk, CT) spectrophotometer. $10 \mu \mathrm{L}$ of a matrix solution $(0.11 \mathrm{M}$ in methanol) was deposited onto the interior of a $1-\mathrm{cm}$ methacrylate cuvette. A methacrylate cuvette was used because it is transparent in the range of wavelengths being scanned. Absorption spectra $(245-600 \mathrm{~nm})$ were acquired from the resultant dry thin film. The fully illuminated area of each matrix thin film was approximately $1.0 \times 1.0 \mathrm{~cm}$. 
Luminescence spectra were acquired with an SLM Aminco (SLM Instruments, Inc., Urbana, IL) 8000 fluorometer. Thin films of the matrix compounds were prepared by depositing $10 \mu \mathrm{L}$ of a $0.11-\mathrm{M}$ methanol solution onto a $0.1-\mathrm{mm}$-thick methacrylate slide. The slide was then inserted diagonally into a methacrylate cuvette and placed in the fluorometer's sample holder for subsequent spectral acquisition. A background fluorescence spectrum was taken with an uncoated methacrylate slide in place, and the background spectrum was subtracted from each matrix emission spectrum. The illuminated area of each thin film was approximately $1.0 \times 1.0 \mathrm{~cm}$. The excitation was manually set at $4-\mathrm{nm}$ spectral line width. The excitation wavelength used for the measurements was $337( \pm 4) \mathrm{nm}$. The luminescence signal was measured at the $\lambda_{\max }$ emission for each matrix studied.

\section{Calculations of Matrix Acidities}

Semiempirical calculations were performed with MOPAC version 6.0 (CaChe Worksystem 3.0, CaChe Scientific, Beaverton, OR) on a MacIntosh (Apple Computer Co., Inc., Cupertino, CA) Quadra 900. Starting geometries were obtained with the Molecule Editor program incorporated in the CaChe Worksystem. Calculations were performed with the standard PM3 program based on the restricted Hartree-Fock method. Geometries were optimized by using analytical gradients, and the search was terminated when the change in energy upon successive iterations was less than $1 \times 10^{-9} \mathrm{kcal} / \mathrm{mol}$. The heats of formation (enthalpies) of the heavy-atom-substituted salicylic acids and their corresponding anions were calculated for the ground $\left(S_{0}\right)$ and first excited $\left(S_{1}\right)$ states. The calculated energy difference between the neutral and corresponding anion is referred to as the enthalpy of proton transfer or $\Delta H$. The $\Delta H$ is proportional to the Gibbs free energy of reaction $(\Delta G)$, and thus proportional to $\mathrm{p} K_{a}$, because the contribution of $\Delta S$ is small. Calculations were performed with full optimization of all geometrical variables (bond lengths, bond angles, and dihedral angles) with no symmetry constraint. The conformation with the lowest energy was selected as optimum.

\section{Results}

In initial attempts to measure absorbance and luminescence of matrix films, quartz cuvettes were used; however, the thin films prepared on quartz dried unevenly and either developed cracks or peeled. It was found that films produced on methacrylate were much more uniform in thickness and gave reproducible absorbance and luminescence results. Table 1 contains solid-phase absorption and measured luminescence (unresolved fluorescence and phosphorescence) data compiled from four replicate runs for each matrix thin film on methacrylate. The tabulated data include average measured absorbance at $337 \mathrm{~nm}$ (the wavelength of the $\mathrm{N}_{2}$ laser used in the MALDI experiments), average measured luminescence (by using $337-\mathrm{rm}$ excitation), and the average percent decrease in luminescence observed for the heavy-atom-substituted compound relative to the corresponding unsubstituted compound. The standard deviation for each value is listed as well. We have attempted to maintain the surface area and amount of matrix constant by applying the same volume of each matrix solution to the methacrylate cuvette or slide. It is not, however, the purpose of this study to provide a quantitative measurement of the solid-state absorbance and luminescence of the matrices studied, but to illustrate the qualitative trends. Additional absorption and luminescence experiments were performed where analyte was added to the matrix solutions prior to deposition on the methacrylate cuvette or slide. No detectable difference (within experimental error) was observed in the absorption and emission spectra of the matrix films to which analyte was added.

Comparison of the measured absorbance of the matrix pairs at $337 \mathrm{~nm}$ (Table 1) shows considerable variation. Measured luminescence intensities also vary widely among the different compound classes (e.g.,

Table 1. Comparison of absorption and luminescence spectral data from heavy-atom-substituted and nonsubstituted matrices (excitation wavelength $=337 \mathrm{~nm}$ )

\begin{tabular}{|c|c|c|c|}
\hline Matrix & $\begin{array}{c}\text { Absorbance } \\
\text { (337 nm })\end{array}$ & $\begin{array}{c}\text { Measured } \\
\text { Luminescence }\end{array}$ & $\begin{array}{l}\text { Lum. Decrease with } \\
\text { Heavy-Atom Subs. (\%) }\end{array}$ \\
\hline 4-Hydroxy-3-nitrobenzoic acid & $0.36( \pm 0.07)$ & $3150( \pm 207)$ & \\
\hline 4-Hydroxy-3-iodo-5-nitrobenzoic acid & $0.14( \pm 0.03)$ & $2222( \pm 149)$ & $29( \pm 3)$ \\
\hline 2,4-Dinitroaniline & $0.42( \pm 0.05)$ & $2207( \pm 329)$ & \\
\hline 2-Broma-4,6-dinitroaniline & $0.40( \pm 0.05)$ & $1736( \pm 180)$ & $21( \pm 4)$ \\
\hline 2,4-Dihydraxybenzoic acid & $0.021 \pm 0.0061$ & $5680( \pm 724)$ & \\
\hline 5-Bromo-2,4-dihydroxy-benzoic acid & $0.041 \pm 0.0031$ & $2845( \pm 762)$ & $50( \pm 15)$ \\
\hline Salicylic acid & $0.05( \pm 0.009)$ & $5960( \pm 327)$ & \\
\hline 5-Fluorosalicylic acid & Not measured & $5809( \pm 460)$ & $3( \pm 0.3)$ \\
\hline 5-Chlorosalicylic acid & Not measured & $5232( \pm 610)$ & $12( \pm 1.4)$ \\
\hline 5-Bromasalicylic acid & Not measured & $2627 \pm 1380)$ & $56( \pm 30)$ \\
\hline 5-lodosalicylic acid & $0.27( \pm 0.05)$ & $872( \pm 350)$ & $85( \pm 34)$ \\
\hline
\end{tabular}




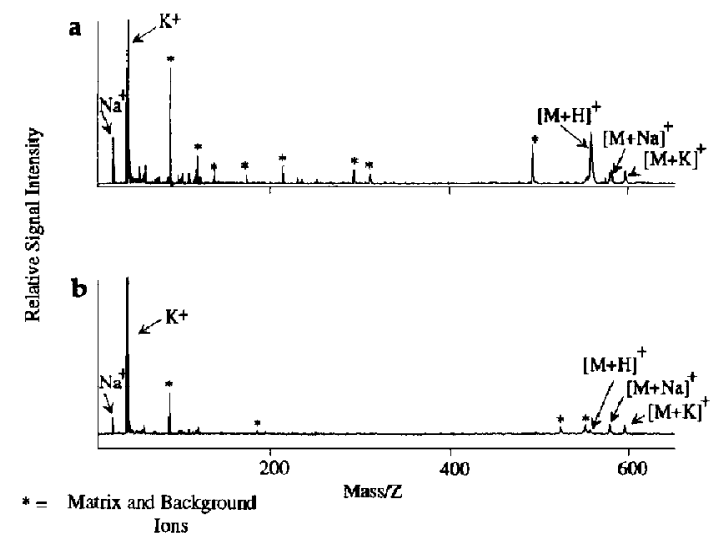

Figure 1. Positive ion MALDI mass spectra of Leu-enkephalin obtained (a) with 4-hydroxy-3-iodo-5-nitrobenzoic acid and (b) with 4-hydroxy-3-nitrobenzoic acid. Peaks labeled with an asterisk are matrix ions. The matrix-to-analyte ratio $(M-A)$ with both matrices is $120: 1$. Conditions: $V_{a c c}=10 \mathrm{kV}$; spectra averaged over 100 laser shots. The scale for the $y$-axis of spectrum (a) and spectrum (b) is the same.

nitro-aromatic versus carboxylic acid-aromatic), but in all cases addition of a heavy-atom (bromine or iodine) resulted in a significant decrease in the measured luminescence. There is no corresponding correlation for absorption data.

Figure 1 contains MALDI mass spectra of Leu-enkephalin obtained by using 4-hydroxy-3-iodo-5-nitrobenzoic acid and 4-hydroxy-3-nitrobenzoic acid as matrices. The Leu-enkephalin $[\mathrm{M}+\mathrm{H}]^{+}$ion yield (calculated by using integrated peak areas) is about five times greater with the use of the iodo-substituted matrix than with 4-hydroxy-3-nitrobenzoic acid. Comparisons of Leu-enkephalin $[\mathrm{M}+\mathrm{H}]^{+}$ion yield were made for three other pairs of heavy-atom-substituted and nonsubstituted matrices. Table 2 contains data for the Leu-enkephalin $[\mathrm{M}+\mathrm{H}]^{+}$ion yields obtained by using these compounds, as well as the estimated standard deviation in ion yield, calculated from multiple sets of data. Note that the heavy-atom compounds consistently produce a greater Leu-enkephalin [M+ $\mathrm{H}]^{+}$ion yield than the nonheavy-atom-substituted compounds.

Leu-enkephalin was chosen as the probe analyte, rather than a larger species, to allow complete resolution of the $[\mathrm{M}+\mathrm{H}]^{+}$ion from any adduct or matrix ions. In particular, we wanted to verify that the changes in ion yield actually corresponded to a change in the abundance of the $[\mathrm{M}+\mathrm{H}]^{+}$ion as opposed to changes in the abundances of the $[\mathrm{M}+\mathrm{Na}]^{+},[\mathrm{M}+\mathrm{K}]^{+}$, and /or $[\mathrm{M}+\text { matrix adduct }]^{+}$ions. The mass resolution of the linear TOF mass spectrometer used in these experiments is not sufficient to resolve the $[\mathrm{M}+\mathrm{H}]^{+}$ion of higher molecular weight analytes (e.g., myoglobin) from adduct ions. Note, however, that there is an enhancement of total analyte ion yield for myoglobin obtained by using heavy atom matrices. For example, the ion yield for myoglobin obtained by using 4-hy-
Table 2. Comparison of Leu-enkephalin ion signal observed by using heavy-atom-substituted and nonsubstituted matrices

\begin{tabular}{lc}
\hline Matrix & {$[\mathrm{M}+\mathrm{H}]^{+}$Ion Yield $^{\mathrm{a}}$} \\
\hline \hline 4-Hydroxy-3-nitro-benzoic acid & $0.27( \pm 0.12)$ \\
4-Hydroxy-3-iodo-5-nitrobenzoic acid & $1.49( \pm 0.88)$ \\
Salicylic acid & $0.04( \pm 0.01)$ \\
5-lodosalicylic acid & $0.34( \pm 0.02)$ \\
2,4-Dinitroaniline & $0.82( \pm 0.21)$ \\
2-Bromo-4,6-dinitroaniline & $2.20( \pm 0.52)$ \\
2,4-Dihydroxybenzoic acid & $0.05( \pm 0.02)$ \\
5-Bromo-2,4-dihydroxy-benzoic acid & $0.16( \pm 0.06)$ \\
\hline
\end{tabular}

${ }^{\mathrm{A}}[\mathrm{M}+\mathrm{H}]^{+}$ion yield is based on peak area lunits are in millivolts over timel.

droxy-3-iodo-5-nitrobenzoic acid is approximately 1.5 times greater than that observed by using 4-hydroxy3-nitrobenzoic acid.

MALDI mass spectra of vitamin $B_{12}$ also were obtained by using the same series of matrix compounds. Figure 2 contains MALDI mass spectra of vitamin $B_{12}$ acquired by using 4-hydroxy-3-iodo-5-nitrobenzoic acid and 4-hydroxy-3-nitrobenzoic acid. The spectra from both matrices contain signals at $m / z 1356\left([\mathrm{M}+\mathrm{H}]^{+}\right)$, $1329\left([\mathrm{M}+\mathrm{H}-\mathrm{CN}]^{+}\right)$, and 971 (loss of $\mathrm{CN}$, base, sugar, and phosphate groups from the vitamin $B_{12}$ ). The relative abundance of these ions differs substantially depending upon the matrix [26]. The $m / z 971$ fragment ion is the dominant ion in the spectrum obtained by using 4-hydroxy-3-nitrobenzoic acid, whereas the dominant ion in the spectrum taken by using 4-hydroxy-3-iodo-5-nitrobenzoic acid is the [M $+\mathrm{H}]^{+}$ion. Another difference in the two spectra is the overall amount of fragmentation observed. The spectrum acquired by using the iodo-substituted matrix contains little fragmentation beyond the ions at 971

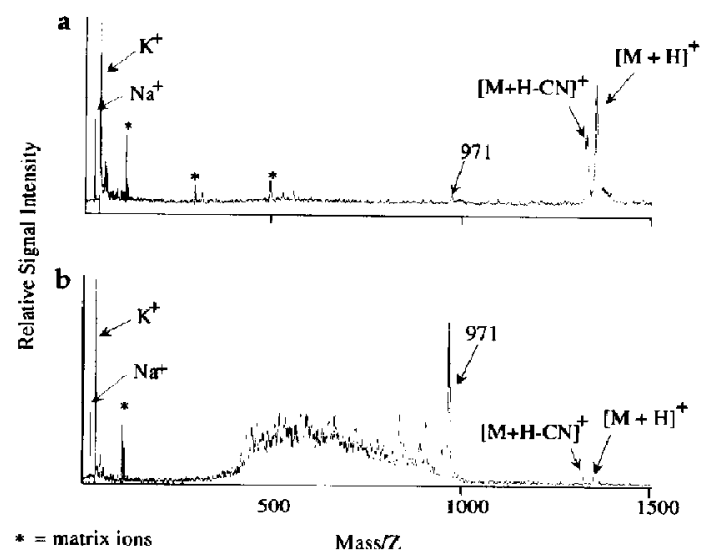

Figure 2. Positive ion MALDI mass spectra of vitamin $B_{12}$ obtained (a) with 4-hydroxy-3-iodo-5-nitrobenzoic acid and (b) with 4-hydroxy-3-nitrobenzoic acid. Peaks labeled with an asterisk are matrix ions. The matrix-to-analyte ratio (M-A) with both matrices is 120:1. Conditions: $V_{\text {acc }}=10 \mathrm{kV}$; spectra averaged over 100 laser shots. The scale for the $y$-axis of spectrum (a) and spectrum (b) is the same. 
and $1329 \mathbf{u}$, whereas the spectrum acquired with the nonsubstituted matrix contains abundant fragment ions in the region below 971 u. Similar fragment ions are observed for vitamin $B_{12}$ by using ${ }^{252}$ Cf-plasma desorption mass spectrometry [27] and $\mathrm{Cs}^{+}$ion secondary ionization mass spectrometry (by TOF [28] and Fourier-transform ion cyclotron resonance (FTICR) [29, 30]). We also examined the laser power dependence for the analyte $[\mathrm{M}+\mathrm{H}]^{+}$ion yield versus fragmentation for the heavy-atom matrices. Even at laser powers an order of magnitude greater than threshold, the abundance of the vitamin $B_{12}$ fragment ions obtained by using 4-hydroxy-3-iodo-5-nitrobenzoic acid is much less than that obtained by using $\alpha$-cyano- 4 -hydroxycinnamic acid [26].

Table 3 lists the vitamin $\mathrm{B}_{12}[\mathrm{M}+\mathrm{H}]^{+}$ion yield obtained by using each of the heavy-atom and nonheavy-atom matrices. The heavy-atom matrices consistently yield a greater abundance for the $[\mathrm{M}+\mathrm{H}]^{+}$ion than the nonsubstituted matrices. Note that for the salicylic acid derivatives the measured luminescence (Table 1) decreases as the mass of the heavy-atom substituents increases, whereas the vitamin $B_{12}[M+$ $\mathrm{H}^{+}$ion yield increases.

\section{Discussion}

The most important observation from this study is the correlation between MALDI analyte ion yield and changes in measured luminescence (and therefore relaxation dynamics) of the heavy-atom-substituted and nonsubstituted matrix pairs. Incorporation of a heavy atom into the matrix promotes intersystem crossing [e.g., singlet to triplet transitions $\left(S_{1} \rightarrow T_{1}\right)$ ]. Such a decay route could affect MALDI ion yields in two ways:

1. If proton transfer occurs exclusively from $S_{1}$, then the MALDI ion yield-specifically $[\mathrm{M}+\mathrm{H}]^{+}$ion yield-would decrease upon heavy-atom substitution of a matrix.

Table 3. Comparison of ion signal observed for vitamin $B_{12}$ using heavy-atom-substituted and nonsubstituted matrices

\begin{tabular}{ll}
\hline Matrix & $\begin{array}{c}{[\mathrm{M}+\mathrm{H}]^{+} \text {lon }} \\
\text { Yield }^{\mathrm{a}}\end{array}$ \\
\hline \hline 4-Hydraxy-3-nitro-benzoic acid & $0.02( \pm 0.006)$ \\
4-Hydroxy-3-iodo-5-nitrobenzoic acid & $0.67( \pm 0.064)$ \\
Salicylic acid & 0 \\
5-Fluorosalicylic acid & 0 \\
5-Chlorosalicylic acid & $0.01( \pm 0.003)$ \\
5-Bromosalicylic acid & $0.03( \pm 0.007)$ \\
5-Iodosalicylic acid & $0.05( \pm 0.009)$ \\
2,4-Dinitroaniline & 0 \\
2-Bromo-4,6-dinitroaniline & $0.29( \pm 0.042)$ \\
2.4-Dihydroxybenzoic acid & 0 \\
5-Bromo-2,4-dihydroxy-benzoic acid & $0.15( \pm 0.042)$ \\
\hline
\end{tabular}

a Vitamin $B_{13}[M+H]^{+}$ion yield based on calculated peak area (units are in millivalts over time).
2. If proton transfer occurs from either $S_{1}$ or $T_{1}$, but is dependent on the lifetime of the excited state, then heavy-atom substitution would increase analyte [M] $+\mathrm{H}]^{+}$ion yield because of the longer lifetime of the triplet state.

One important question to address is whether the increased $[\mathrm{M}+\mathrm{H}]^{+}$ion yield observed for heavyatom- versus nonheavy-atom-substituted matrices is because of changes in the absorbance at $337 \mathrm{~nm}$. That is, do matrices that absorb more strongly at $337 \mathrm{~nm}$ result in a greater analyte ion yield? If this were true, 4-hydroxy-3-nitrobenzoic acid should produce a greater abundance of $\left[\mathrm{M}+\mathrm{H}^{+}\right.$ions than 4-hydroxy-3-iodo5-nitrobenzoic acid. However, the Leu-enkephalin [M $+\mathbf{H}]^{+}$ion yield is five times greater by using 4-hydroxy-3-iodo-5-nitrobenzoic acid than for 4-hydroxy3-nitrobenzoic acid (see Table 2). Clearly, there appears to be no correlation between $[\mathrm{M}+\mathrm{H}]^{+}$ion yield and matrix absorbance at $337 \mathrm{~nm}$. In a separate study we measured laser energy thresholds for MALDI ion production for several matrices (e.g., $\alpha$-cyano-4-hydroxycinnamic acid, 4-nitroaniline, and 7-hydroxy-4-methylcoumarin) and found that the threshold for ion production does not correlate with absorbance at $337 \mathrm{~nm}$ (Preston-Schaffter, L. M.; Russell, D. H., manuscript in preparation). Our current investigations are focused on determining matrix characteristics other than absorption (e.g., fluorescence, matrix-analyte interactions) that play a role in determining the MALDI threshold.

The decreased luminescence intensity observed for the heavy-atom-substituted matrix compounds is consistent with the view that the $S_{1}$ state is depopulated by nonradiative processes, possibly intersystem crossing $[23,24]$. We argue that decay of the nonheavy-atom $S_{1}$ species can follow two routes: (1) radiative decay (primarily fluorescence) and (2) nonradiative decay (internal conversion and/or proton transfer). For proton transfer to be competitive with fluorescence it must take place on a time scale comparable to the excited state lifetime. The observation by Chiarelli et al. [7] that salicylic acid has a longer fluorescence lifetime than $p$-hydroxybenzoic acid and more readily undergoes excited state proton transfer is consistent with this idea. Proton transfer on the nanosecond time scale requires intimate contact between the matrix and analyte (e.g., direct interaction via hydrogen bonding) prior to photoexcitation. This suggestion is consistent with Beavis and Bridson's [31] model of inclusion of the analyte in the matrix crystal lattice, that is, MALDI requires intimate contact between the matrix and analyte. Alternatively, the probability of proton transfer might be increased if the lifetime of the "photochemically activated" matrix, (matrix)*, were extended. This latter mechanism would be achieved by promoting intersystem crossing of (matrix)* to the triplet manifold $\left(S_{1} \rightarrow T_{1}\right)$ [32] and would explain the observed decrease in measured luminescence and increased analyte $[\mathrm{M}+\mathrm{H}]^{+}$ion yields obtained when heavy-atom matrices are used in MALDI. However, regardless of 
the specific mechanism for ion formation, the correlation between changes in relaxation dynamics of matrices (as measured by changes in measured luminescence) and analyte $[\mathrm{M}+\mathrm{H}]^{+}$ion yields is consistent with the involvement of excited state processes in MALDI.

Another factor to consider is whether heavy-atom substitution changes the acidity of the matrix compound. MOPAC semiempirical calculations were used to estimate the ground and excited state acidities of unsubstituted and heavy-atom-substituted salicylic acid matrices. On the basis of these calculations, we conclude that heavy-atom substitution does not cause a significant change in the acidities of the $S_{1}$ or $S_{0}$ states of aromatic alcohols, which is consistent with data reported by Ireland and Wyatt [16]. That is, the polarization of these compounds is dominated by the strong electron-releasing or -withdrawing groups such as $-\mathrm{NH}_{2},-\mathrm{OH},-\mathrm{NO}_{2}$, or $-\mathrm{COOH}$ substituents [33] and the heavy-atom substituents have very little effect on the gas-phase acidities.

In a previous paper, we argued that the fragmentation of vitamin $\mathrm{B}_{12}[\mathrm{M}+\mathrm{H}]^{+}$ions by using various matrices was dependent on the enthalpy of the proton transfer reaction, that is, on the relative acidities of the matrices [26]. Because the heavy- and nonheavy-atom matrices used here have similar acidities (see above) the greater amount of fragmentation observed for vitamin $B_{12}$ (relative to $\left[\mathrm{M}+\mathrm{H}^{+}\right.$ions) with nonheavyatom matrices must involve other factors in addition to matrix acidity. For example, proton transfer reactions that involve the heavy-atom-substituted matrix may occur from the $T_{1}$ species, which lies lower in energy than the $S_{1}$ state, so that less energy is available for partitioning as internal energy into the analyte. Although this seems a logical argument because heavyatom substitution promotes internal conversion to the triplet state $[23,24]_{s}$ it does not explain why the excess ro-vibronic energy present in $T_{1}$ is not partioned as internal energy of the analyte ion thereby leading to fragmentation. Independent of the exact details of the ion formation process, these results clearly show that the yield of $[\mathrm{M}+\mathrm{H}]^{+}$and fragment ions can be "tuned" by judicious choice of the matrix. Additional studies with the heavy-atom matrices are under way. These studies focus on energy transfer between the matrix and analyte and the possible additional role of internal conversion in the energy transfer process.

\section{Conclusion}

The results of this study clearly implicate the involvement of excited state processes in the proton transfer reaction that leads to analyte ionization in MALDI. This conclusion is based on the fact that perturbing the excited state of a matrix with a heavy-atom substituent causes a direct response in the MALDI analyte [M + $\mathrm{H}^{+}$ion yield. The heavy-atom experiments demonstrate the utility of probing the effects of matrix structure (e.g., electronic structure and relaxation dynamics) on MALDI analyte ion yields and suggest that future studies that examine other aspects of matrix structure (e.g., acidity) may lead to further insight into the merhanism of ion formation. The information obtained from these types of studies could be further enhanced by time-resolved luminescence studies (separating phosphorescence from fluorescence) and more detailed probes of the role of excited state processes in MALDI.

\section{Acknowledgments}

We wish to thank Dr. Paul Lindahl from the Texas A\&M University Department of Chemistry for the use of his PerkinElmer Lambda 3B spectrophotometer and Dr. Tom Baldwin from the Texas A\&M University Department of Biochemistry/Biophysics for the use of his SLM Aminco 8000 fluorometer. We would also like to thank Mary E. Gimon from the Texas A \& M University Department of Chemistry for performing the semiempirical calculations to determine the ground and excited state gas-phase acidities of the heavy-atom-substituted salicylic acid matrices. This research was supported by grants from the U.S. Department of Energy, Division of Chemical Science, Office of Basic Energy Sciences (DE-FG05-85-ER13434) and the National Science Foundation (CHE-8821780). Funds to build the mass spectrometer used in this research were provided by the Center for Macromolecular Design, Texas A \& M University, College of Science Research Enhancement Fund, and the Department of Chemistry, Texas A \& M University.

\section{References}

1. Karas, M.; Bachmann, D.; Bahr, U.; Hillenkamp, F. Int. I. Mass Spectrom. Ion Processes 1987, 78, 53-68.

2. Tanaka, K.; Waki, H.; Ido, Y.; Akita, S.; Yoshida, Y.; Yoshida, T. Rapid Commun. Mass Spectrom. 1988, 2, 151-153.

3. Vertes, A.; Gijbels, R.; Levine, R. D. Rapid Commun. Mass Spectrom. 1990, 4, 228-233.

4. Vertes, A.; Gijbels, R. Scanning Microscopy 1991, 5, 9344-9363.

5. Sundqvist, B.; Hedin, A.; Håkansson, P.; Jonsson, G.; Salehpour, M.; Säve, G.; Widdiyasekera, S.; Roepstorff, P. IFOS III Springer Proceedings in Physics; 1986; pp 7-10.

6. Johnson, R. E.; Sundquist, B. U. R. Rapid Commun. Mass Spectrom. 1991, 5, 574-578.

7. Chiarelli, M. P.; Sharkey, Jr., A. G.; Hercules, D. M. Anal. Chem. 1993, 65, 307-311.

8. Ehring, $\mathrm{H}_{;}$K Karas, M, Hillenkamp, F, Org. Mass Spectrom. $1992,27,472-480$.

9. Gimon, M. E.; Preston, L. M.; Solouki, T.; White, M. A.; Russell, D. H. Org. Mass Spectrom. 1992, 27, 827-830.

10. Wang, B. H.; Dreisewerd, K.; Bahr, U.; Karas, M.; Hillenkamp, F. J. Am. Soc. Mass Spectrom. 1993, 4, 393-398.

11. Levis, R. J.; Romano, L. J. I. Am. Chem. Soc. 1991, 113 $7802-7803$

12. Romano, L. J.; Levis, R. J. I. Am. Chem. Soc. 1991, 113, $9665-9667$.

13. Speir, J. P.; Amster, I. J. Anal Chem. 1992, 64, 1041-1045.

14. Juhasz, P.; Costello, C. E.; Biemann, K. J. Am. Soc. Mass Spectrom. 1993, 4, 399-409.

15. Förster, Th. Pure Appl. Chem. 1973, 34, 225-234.

16. Ireland, J. F.; Wyatt, P. A. H. Adv. Phys. Org. Chem. 1976, 12, 131-221.

17. Huppert, D.; Gutman, M.; Kaufmann, K. J. Adv. Chem. Phys. $1980,47,643-679$. 
18. Davidson, R. S. In Advances in Physical and Organic Chemistry, Vol. 19; Gold, V.; Bethell, D., Eds.; Academic Press: London, 1983; pp 1-130.

19. Creed, D. Photochem. and Photobiol. 1984, 39. 537-562.

20. Ireland, J. F.; Wyatt, P. A. H. J. Chem. Soc. Faraday I 1972, 68, 1053-1058.

21. Parker, C. D.; Hercules, D. M. Anal. Chem. 1986, 58, 25-30.

22. Chiarelli, M. P.; Gross, M. L. J. Phys. Chem. 1989, 93, 3595-3599.

23. Kasha, M. J. J. Chem. Phys. 1952, $20,71$.

24. Purdy, B. B.; Hurtubise, R. I. Appl. Spectrosc. 1992, 46, 988-993.

25. Preston, L. M.; Murray, K. K.; Russell, D. H. Biol. Mass Spectrom. 1993, 22, 544-550.
26. Kinsel, G. R.; Preston-Schaffter, L. M.; Russell, D. H. Biol. Mass Spectrom., 1994, 23, 205-211.

27. Macfarlane, R. D.; Torgerson, D. F. Science 1976, 191, 920.

28. Ens, W.; Standing, K. G.; Chait, B. T.; Field, F. H. Anal. Chem. 1981, 53, 1241.

29. Castro, M. E.; Russell, D. H. Anal. Chem. 1984, 56, 578.

30. Solouki, T.; Russell, D. H. Appl. Spectrosc. 1993, 47, 211-217.

31. Beavis, R. C.; Bridson, J. N. J. Phys. D 1993, 26, 442-447.

32. Schulman, S. G. Fluorescence and Phosphorescence Spectroscopy: Physicochemical Principles and Practice, Pergamon Press; New York, 1977, pp 66-95.

33. Hansch, C.; Leo, A,j Taft, R. W. Chem. Rev. 1991, 91, 165-175. 